\title{
Myocardial infarction in non-dissecting aortic root aneurysm
}

\author{
Abdallah K. Alameddine ${ }^{1,2^{*}}$, Richard J. Hicks ${ }^{2,3}$, Victor Alimov ${ }^{1,2}$, Yvonne A. Alameddine ${ }^{1}$, \\ Joseph E. Flack ${ }^{1,2}$ \\ ${ }^{1}$ Division of Cardiac Surgery, Baystate Medical Center, Springfield, USA \\ ${ }^{2}$ The Tufts School of Medicine, Boston, USA \\ ${ }^{3}$ The Department of Radiology, Baystate Medical Center, Springfield, USA \\ Email: akalameddine@gmail.com
}

Received 16 January 2013; revised 15 March 2013; accepted 19 April 2013

Copyright (c) 2013 Abdallah K. Alameddine et al. This is an open access article distributed under the Creative Commons Attribution License, which permits unrestricted use, distribution, and reproduction in any medium, provided the original work is properly cited.

\section{ABSTRACT}

We describe a case of a 49-year-old man who presented with an uncomplicted aortic root aneurysm, aortic insufficiency, and ST-elevation myocardial infarction (STEMI) without obstructive coronary artery disease on angiography. The computed tomography angiogram (CTA) of the thorax, performed without cardiac gating, was misinterpreted as normal. In retrospect, an overlooked extravasation of contrast material lateral to the aortic root was detected on non-gating magnetic resonance angiography (MRA). Exploration of the aortic root revealed an unsuspected horizontal intimal tear of the left sinus of Valsalva with limited extramural hematoma. The presence of an otherwise silent intimal tear on preoperative imaging studies makes the overall management more problematic. For example, initiating early broad empirical anticoagulants or fibrinolytics therapy to treat the accompanied myocardial infarction may extend the tear into a full life-threatening aortic dissection, tamponade or rupture. We highlight many of the difficulties associated with the diagnosis and treatment of limited sinus tear when aortic root aneurysm is presenting with cryptogenic STEMI. Accurate morphologic characterization of intimal tear would be best defined with either an electrocardiogram-gating CTA or MRA imagings. These non-invasive tests are needed to make appropriate management decisions. Depending on other pathologic components of aortic root, cusps and the commissural geometry, sinus tear is a critical component for the overall treatment plan and it shifts the surgical intervenetion from valvesparing operation, commissural resuspension and leaflet repair to composite aortic root replacement (modi-

*Corresponding author. fied version of the Bentall procedure).

Keywords: Myocardial Infaction; Valsalva Sinus Tear; Aortic Root Aneurysm

\section{INTRODUCTION}

The diagnosis and management of ST-elevation myocardial infarction (STEMI) are well known, whereas in patients presenting with asymptomatic aortic root aneurysms, management of STEMI in the setting of aortic sinus intimal tear is much more challenging. Unlike dissection, limited intimal tears have an exposed subintimal layer without major flap or significant hematoma formation, features that might evade preoperative imaging studies such as computed tomographic angiography (CTA). The overwhelming number of patients with STEMI will indeed have associated coronary pathology. We describe our experience with an illustrative case of STEMI with normal coronary angiogram, and non-dissecting aortic root aneurysm. Then we discuss the differential diagnoses that may lead to such a scenario. In this context, it is vital to consider aortic sinus tear as a likely source that may lead to STEMI. Furthermore, not only the treatment for the STEMI in the presence of sinus tear differ, but also the choice of reparability techniques of the aortic valve and the associated root aneurysm is influenced by the presence or absence of any sinus pathology that may affect the aortic root morphology [1,2]. The following case points up some of these diagnostic and treatment considerations and the dilemma that the practicing surgeon interested in aortic surgery might face.

\section{CLINICAL SUMMARY}

A 49-year-old male patient with medical history of pro- 
gressive congestive heart failure (CHF), hypertension, hyperlipidemia and episodic atrial fibrillation, presented to the emergency room with substernal chest pain and diaphoresis that had first appeared intermittently 2 weeks earlier but had become markedly intense within the past few hours. The patient had a family history of type B aortic dissection. There was no personal history of thrombotic disorders, no recent febrile infections, and the patient did not have a history of cigarette smoking, chronic alcoholism or use of recreational drugs. His medications on arrival included hydrochlorothiazide, valsatran and verapamil extended release. He was obese with a body-mass index of $32 \mathrm{~kg} / \mathrm{m}^{2}$. He had normal equal blood pressures in both arms and the chest evaluation revealed a faint murmur difficult to evaluate because of a thick chest wall. A 12-lead electrocardiogram showed STsegment elevations up to $4 \mathrm{~mm}$ in leads II, III, V5, V6, and AVF. The chest radiograph was normal. The levels of serum cardiac troponin (cTnT) was 1.77 ng per milliliter (reference range, $<0.09$ ), and the level of $\mathrm{MB}$ isoenzyme creatine kinase was 83.9 ng per milliliter (reference range, $<6$ ). Cardiac catheterization obtained within 8 hours after the onset of chest pain showed non-obstructive coronary arteries with absence of thrombosis or spasm. The left ventriculogram revealed a $5.5 \mathrm{~cm}$ aortic root aneurysm and moderate to severe aortic insufficiency (AI) $(3 / 4+)$. His symptoms resolved over period of few hours after admission, with beta-blockers and angiotensin-converting enzyme for blood pressure control along with oxygen, aspirin, morphine and a 5000-U bolus of intravenous heparin. Continuous infusion of heparin and nitroglycerin was initiated.

Transthoracic and esophageal echocardiography, CTA and MRA imagings all confirmed the presence of an uncomplicated aortic root aneurysm and severe aortic insufficiency (AI) with an eccentric jet. The ejection fraction was $60 \%$. On the basis of the information we had, an acute event in the dilated aortic root seemed to be the most likely predisposing event for both the STEMI and severe AI. In the absence of a plausible etiology of the MI, and because of the degree of AI, an urgent surgery was decided despite the perceived clinical and hemodynamic stability. Exploration via median sternotomy revealed $300-400 \mathrm{ml}$ of bloody pericardial fluid and a trileaflet aortic valve with prominent fenestrations of the right and left coronary leaflets. The left coronary leaflet had a small perforation in it, and above the leaflet there was an unanticipated curvilinear (2 cm length) intimal tear that spared the coronary osteum. Associated with it, there was a small mediastinal hematoma (walnut size) extramural to the aneurysm wall with exposed subintimal tissue but without significant separation. No vegetations or thrombi were noted, and gram stain was negative for bacteria. A composite aortic graft using 27 Magna Ease (Edwards Lifesciences, Irvine, CA) pericardial valve (patient's choice) and a $30 \mathrm{~mm}$ sinus of Valsalva graft (St. Jude Medical, St. Paul, Minnesota), were sewn in place to reconstruct the aortic root. The left main coronary artery was encased within thickened tissues adjacent to the tear area that was unappreciated on the intial CTA or MRA imagings. Through the left atrial dome, a left atrial Cryomaze procedure completed the operation. His postoperative course was uneventful and he went home on postoperative day 9. Histology of the aortic wall showed minimal amount of atherosclerotic plaque.

In retrospect the original $3 \mathrm{~mm}$ thick source image from CTA demonstrated a $3 \mathrm{~cm}$ diameter abnormal contrast collection, adjacent to the aortic root. No intimal flap was present. The contrast collection may have been misinterpreted as a portion of the left atrium but this clearly seen posterior to the extravasation (Figure 1(a)). The coronal reconstruction, part of the original examination, much better shows the perforation (Figure 1(b)), but this was again overlooked or misidentified. Gadolinium enhanced MRA of the chest was performed after the CTA and acquired in an angled sagittal plane parallel to the aortic arch, showed extravasation of contrast medium, extending laterally from the aortic root (Figure 2(a)) that was also overlooked, likely due to its lack of continuity with the aortic root in this plane and misidentified as a portion of the left atrium. Coronal reconstructions (Figure 2(b)), not available as part of the original examination, clearly demonstrate the abnormality.

\section{DISCUSSION}

A normal coronary angiography in patients presenting with biochemically confirmed acute ST-elevation myocardial infarction (MI) is extremely rare, (0.7\%) [3]. According to published guidelines, in the initial management of patients with acute ST-segment elevation myocardial infarction, aspirin and heparin or antithrombotic therapy are used [4]. However, management with anticoagulants should be avoided until we have relevant

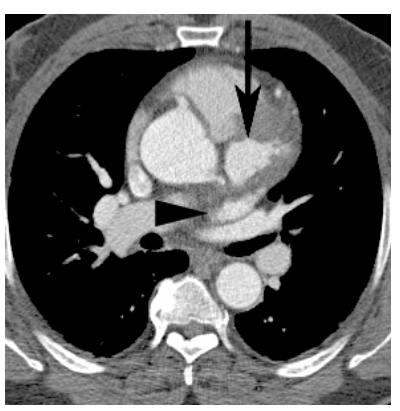

(a)

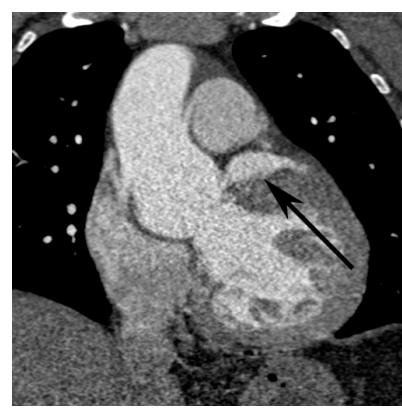

(b)
Figure 1. (a) Axial $3 \mathrm{~mm}$ source image from CTA shows the extravasated contrast (arrow) anterior to the left atrial appendage (arrowhead); (b) Angled coronal reconstruction of CTA dataset reveals contained perforation (arrow) similar to MRA. 


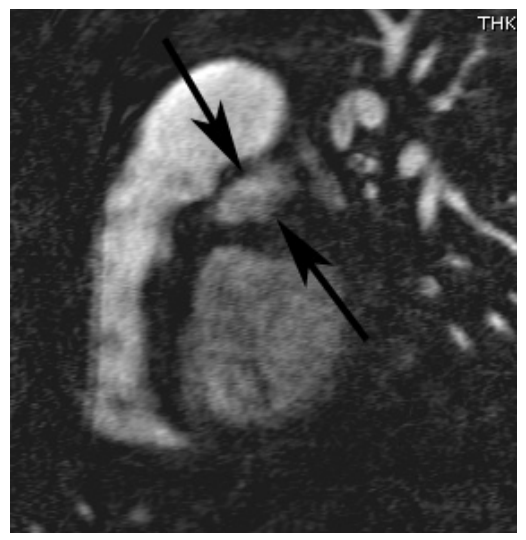

(a)

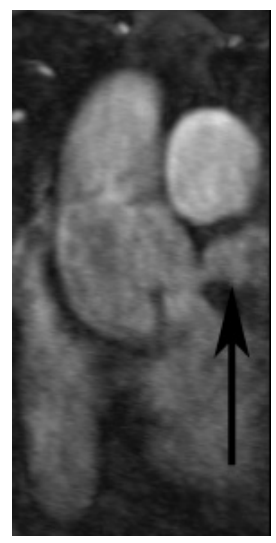

(b)
Figure 2. (a) Gadolinium enhanced angled sagittal MRA reveals abnormal high signal intensity structure (arrows) between the main pulmonary artery and left ventricle; (b) Abnormal collection (arrow) and its relationship to the aortic root is more easily visualized on a coronal reconstruction of the MRA dataset.

studies showing the superiority of not withholding this form of therapy in patients with acute coronary syndrome and aortic dissection (or in intimal tear), for fear of extension of the dissection or for possibly triggering vascular wall hemorrhage and free rupture $[1,2]$. In this patient, in the absence of signs and symptoms suggestive of a dissection, therefore a cardiac catheterization was subsequently performed.

\subsection{The Differential Diagnosis Is Broad}

It was difficult in this case, with the constellation of features to make a unifying diagnosis that connected both findings of aortic root dilation with the STEMI in the absence of coronary artery disease. This patient has risk factors for sinus tear, including hypertension, obesity and a family history of dissection. In aortic dissection, MIs may occur in $4.8 \%$ of patients from blood flow impediment into the coronary arteries by the intimal flap, aortic cusp dehiscence or from luminal compression by the dissecting hematoma [5]. AI in our case can likely be explained by changes of the valve cusps, annular dilation due to aneurysm, or by sinotubular dilation. Contrary to chronic AI, acute AI can be associated with chest pain due to decreased or poor coronary blood flow during diastole [6].

In the absence of aortic dissection, a search for an alternative causes of acute coronary ischemic changes should be sought [7]. The etiology in such a setting is multifactorial [8]. Important causes include anomalous coronary arteries, transient coronary vasospasm (cocaine induced), coronary dissection, hypercoagulability states, coronary inflammation (myocarditis following recent viral infection, or due to vasculitis), and embolization.
Other mechanistic contributors comprise dehisced aortic cusp with coronary obstruction (Venturi effect), the compression and kinking of the left main coronary artery by scar tissue found adjacent to the tear. However, in our case these diagnoses were not supported by the available imaging data. The patient's sinus tear was most consistent cause with the entire clinical picture and it should always be sought after in such a patient.

It is theoretically possible that microthrombi [7] at the tear site may have migrated into the left coronary circulation. Presumably, endothelial tear permits direct blood contact to exposed subintimal collagen resulting in platelets adhesion and aggregation, and initiates the coagulation cascade leading to mural thrombi generation; and the associated tissue destruction can cause the release of procoagulants, such as microparticles and tissue factor will further increase local thrombogenicity . In this patient, pulmonary embolus [9], myocarditis, left ventricular aneurysm, transient coronary vasospasm, and pericarditis should all be considered not likely based on clinical presentation, physical examination, laboratory date and imaging findings.

\subsection{Challenges of Diagnostic Imaging-Studies}

A cardiac catheterization is still necessary for diagnostic purposes. However, in our patient imaging studies did not mesh the limited tear with the anatomic features. Such findings have been reported by Svensson and colleagues [10]. Although in our case the diagnosis of sinus tear was made intraoperatively, the speed of image collection on modern CT equipment makes this imaging technique a useful alternative means of diagnosis. Per our previous protocol, CTAs of the chest, abdomen or pelvis were done without cardiac gating due to the imaging volume required. This resulted in cardiac pulsation artifacts through the aortic root. Despite this artifact, there was good visualization of the sinus of Valsalva and no lesions were detected. We have since modified our protocol to include a repeat ECG-triggered axial CT through the aortic root during a second, smaller, contrast bolus resulting in improved data acquisition of image quality at low radiation [11].

\subsection{What Can We Learn from the Current Case?}

Our case is distinctive because upon reviewing the literature for the past decade we found only a small case series of sinus tear without coronary artery disease nor myocardial infarction, and one case report associated with myocardial infarction without coronary artery disease in which the Valsalva sinus tear has ruptured into the right atrium $[8,10]$. This variant has been named “acute aortic syndrome” [12]. The finding of STEMI with normal coronary angiogram and uncomplicated aor- 
tic root aneurysm should initiate clinical, laboratory, radiologic evaluations to determine the underlying cause. Identification and treatment of an unrevealed sinus of Valsava is crucial. Doing so will greatly reduce the risk of fatal full rupture into the pericardium or the mediastinum and determine the preoperative treatment and surgical planning.

The general message is that the diagnosis of aortic sinus intimal tear, a variant of aortic dissection, can be challenging and should be considered an under recognized underlying disorder of cryptogenic MI; it may be etiologically associated with microthrombi formation and migration into the coronary circulation. Although occult on multiple imaging studies in this case, it is hoped that with better quality cardiac-gated CTA this diagnosis can be made pre-operatively which when recognized changes the treatment approach to the patient. In retrospect, the absence of recurrent acute coronary syndromes during this patient's follow-up, suggests that the intimal tear was the significant factor in precipitating the MI.

\section{CONCLUSION}

The treatment for patients presenting with a non-dissecting ascending aortic aneurysm and that of an associated with "cryptogenic" myocardial infarction differs considerably and the risks and benefits of anticoagulation treatment should be balanced. A young patient with findings of hypertension, family history for dissection and a distinguished aortic regurgitation jet, all of which the current case history implicitly includes, can be synthesized into a final diagnosis of Valsalva intimal tear with limited hematoma formation outside the aortic sinus wall. Any delay in treatment may risk subsequent extension of the tear into a full aortic dissection, free rupture, or cardiac tamponade. Clinical clues and electrocardiographically gated imaging studies that are free of cardiac motion artifact may offer better visualization of sinus tears especially when the aortic root appears normal on the echocardiogram or angiogram. When recognized, the presence of sinus tear changes the approach to the patient. The choice between aortic root replacement or valve sparing procedure can thus be planned depending on the degree of aortic root deformity secondary to the tear.

\section{REFERENCES}

[1] Kamp, T.J., Goldschmidt-Clermont, P.J., Brinker, J.A. and Resar, J.R. (1994) Myocardial infarction, aortic dissection, and thrombolytic therapy. American Heart Journal, 128, 1234-1237. doi:10.1016/0002-8703(94)90756-0
[2] Nallamothu, B.K. and Eagle, K.A. (2002) When zebras run with horses: The diagnostic dilemma of acuteaortic dissection complicated by myocardial infarction. Journal of Interventional Cardiology, 15, 297-299. doi:10.1111/j.1540-8183.2002.tb01107.x

[3] Widimsky, P., Stellova, B., Groch, L., Aschermann, M., Branny, M., Zelizko, M., Stasek, J. and Formanek, P. (2006) Prevalence of normal coronary angiography in the acute phase of suspected ST-elevation myocardial infarction: Experience from the PRAGUE studies. Canadian Journal of Cardiology, 22, 1147-1152. doi:10.1016/S0828-282X(06)70952-7

[4] Antman, E.M., Anbe, D.T., et al. (2011) A report of the American college of cardiology/American heart association task force on practice guidelines (writing committee to revise the 1999 guidelines for the management of patients with acute myocardial Infarction). Journal of the American College of Cardiology, 57, 1920-1959.

[5] Hagan, P.G., Nienaber, C.A., Isselbacher, E.M., et al. (2000) The international registry of acute aortic dissecttion (IRAD): New insights into an old disease. The Journal of the American Medical Association, 283, 897-903. doi:10.1001/jama.283.7.897

[6] Kardasz, I. and De Caterina, R. (2007) Myocardial infarction with normal coronary arteries: A conundrum with multiple aetiologies and variable prognosis: An update. Journal of Internal Medicine, 261, 330-348. doi:10.1111/j.1365-2796.2007.01788.x

[7] Furie, B. and Furie, B.C. (2008) Mechanisms of thrombus formation. The New England Journal of Medicine, 359, 938-949. doi:10.1056/NEJMra0801082

[8] Turgeon, A.F., Dagenais, F., Poirier, P., Bauset, R. and Mathieu, P. (2003) Myocardial infarction in a young woman secondary to a rupture of a noncoronary sinus of Valsalva aneurysm without coronary artery disease. Canadian Journal of Cardiology, 19, 723-724.

[9] Lin, J.F., Li, Y.C. and Yang, P.L. (2009) A case of massive pulmonary embolism with ST elevation in leads $\mathrm{V}$ (1-4). Circulation Journal, 73, 1157-1159. doi:10.1253/circj.CJ-08-0474

[10] Svensson, L.G., Labib, S.B., Eisenhauer, A.C. and Butterly, J.R. (1999) Intimal tear without hematoma: An important variant of aortic dissection that can elude current imaging techniques. Circulation, 99, 1331-1336. doi:10.1161/01.CIR.99.10.1331

[11] Feuchtner, G., Götti, R., Plass, A., Baumueller, S., Stolzmann, P., Scheffel, H., Wieser, M., Marincek, B., Alkadhi, H. and Leschka, S. (2010) Dual-step prospective ECGtriggered 128-slice dual-source CT for evaluation of coronary arteries and cardiac function without heart rate control: A technical note. European Radiology, 20, 20922099. doi:10.1007/s00330-010-1794-7

[12] Lansman, S.L., Saunders, P.C., Malekan, R. and Spielvogel, D. (2010) Acute aortic syndrome. The Journal of Thoracic and Cardiovascular Surgery, 140, S92-S97. 